\title{
Desarrollo de la expresión oral en FLE: la Francofonía y las TIC
}

\section{Development of oral expression in FFL: the Francophonie and ICT}

\author{
$\mathrm{M}^{\mathrm{a}}$ Dolores Asensio Ferreiro \\ Universidad Complutense de Madrid \\ maasen01@ucm.es
}

\begin{abstract}
Communicating effectively and appropriately in a SL implies mastering the four language skills, i.e. written comprehension and expression and oral comprehension and expression. However, not all develop equally during the teaching-learning process in formal contexts. The development of oral expression in FFL is often a challenge for teachers and students due to conditions such as lack of motivation and feelings of inhibition of the students, lack of security or self-confidence. To these are added others such as lack of time, large groups of students or methodological and evaluative gaps that contribute significantly to hinder the practice of this competence in the classroom. This paper presents a teaching project implemented in the FFL classroom where Francophonie, the use of ICT and collaborative learning are key to developing the oral expression of the learner contributing to reinforcing their personal skills.
\end{abstract}

\section{Key-words}

Francophonie, ICT, oral expression, FFL.

\section{Résumé}

Communiquer efficacement et de manière appropriée dans une langue étrangère (LE) implique de maitriser les quatre compétences linguistiques, la compréhension et expression écrites (CE-EE) et la compréhension et expression orales (CO-EO). Cependant, elles ne se développent pas au même niveau au cours du processus d'enseignementapprentissage dans des contextes formels. Le développement de l'expression orale en FLE est souvent un défi pour les enseignants en raison de conditions telles que le manque de motivation et les sentiments d'inhibition de l'élève, le manque de sécurité ou de confiance en soi. À ceux-ci s'ajoutent d'autres comme le manque de temps, de grands groupes d'étudiants ou des lacunes méthodologiques et évaluatives qui réduisent la pratique de cette compétence en classe. Cet ouvrage présente un projet pédagogique mis en oeuvre en classe FLE où la Francophonie, l'utilisation des TIC et l'apprentissage collaboratif sont essentiels pour développer l'expression orale de l'apprenant en même temps qu'elle contribue à renforcer ses compétences personnelles.

Mots-clés

Francophonie, TIC, expression orale, FLE. 


\section{Introducción}

El papel de la comunicación oral como medio de interacción entre los usuarios de una lengua es innegable. De hecho, los seres humanos dedicamos el 75\% de nuestro tiempo a comunicarnos mediante discursos orales. Sin embargo, la expresión oral (EO) es la competencia más compleja para un aprendiente de una LE, todavía más si este aprendizaje debe realizarse en contextos formales. Las exigencias que imponen estos contextos en la práctica docente habitual de una LE implican que la aproximación a la lengua se produzca en condiciones artificiales y, por tanto, provoca que el tratamiento de las competencias no se realice en igualdad de condiciones. Así, el porcentaje dedicado a unas y otras competencias en el aula varía sustancialmente, representando a menudo algunas de ellas algo anecdótico en el proceso de enseñanza-aprendizaje. Este es el caso de la EO. Resulta muy habitual cuestionarse si realmente es posible abordar una competencia tan compleja y difícil como es la expresión oral en el aula. Algunas de las preguntas más recurrentes y polémicas en este sentido suelen ser si se puede aprender a hablar una LE dentro de un aula o, si es posible mejorar esta competencia comunicativa sin estar inmerso en un contexto territorial francófono. Evidentemente, se trata de una cuestión espinosa, ya que cualquier docente de LE reconoce las dificultades que supone abordar esta competencia en el desarrollo de la práctica educativa.

Además de la dificultad de desarrollar la EO en un aula de LE, en el caso del FLE, en un porcentaje muy alto, el alumnado que cursa este idioma, lo hace como segunda lengua extranjera obligatoria (LE2), por detrás de la lengua extranjera internacional por excelencia (LEI), el inglés. Esto condiciona seriamente la enseñanza de esta lengua y de cualquier otra LE2 ya que, a menudo, son percibidas por el alumnado como algo secundario en su currículo y residual para su desarrollo profesional. Es decir, la actitud del alumnado frente al aprendizaje de esta lengua determina en gran medida el éxito o el fracaso del proceso y, por consiguiente, el resultado de dicho aprendizaje. Efectivamente, como define Ajzen (1988: 4), la actitud es la disposición a responder favorable o desfavorablemente a un objeto, una persona, una institución o un evento. Si trasladamos esta definición a las actitudes lingüísticas y, más concretamente, en la lingüística aplicada y la enseñanza de lenguas, encontramos la definición propuesta por Richards, Platt y Platt (1997: 6) que se refiere a las actitudes que los hablantes de diferentes lenguas tienen respecto a las lenguas ajenas. Estos autores aseguran que la expresión de sentimientos positivos o negativos respecto a una lengua puede reflejar impresiones sobre la dificultad o la simplicidad lingüística, la facilidad o dificultad del aprendizaje, el grado de importancia, elegancia, estatus social... y, por tanto, su actitud resulta determinante para su aprendizaje. En esta misma línea, Lasagabaster (2003: 31) sostiene que las actitudes pueden ser tanto un factor de predisposición como un resultado, con lo que un estudiante de una L2 que muestre una actitud positiva hacia el aprendizaje de dicha L2 obtendrá, en la mayoría de los casos, un nivel de competencia en la L2 en cuestión más alto 
que aquel que muestre una actitud más negativa. Todas estas afirmaciones confirman que las actitudes frente al aprendizaje del FLE son determinantes tanto durante el proceso como en el resultado del aprendizaje.

Además de las dificultades de la práctica de la EO en un aula de LE y las actitudes frente a las LE2 como el FLE, se suman otros factores que condicionan seriamente el desarrollo de la EO en el aula de LE2. Uno de ellos se refiere al nivel de L2. Tal y como asegura Alcoba (2000: 124-126), para la puesta en práctica de la EO, el hablante debe contar con un conocimiento de la L2 suficiente para poder producir oraciones y textos y debe poseer la adecuada habilidad y destreza para ser capaz de tomar decisiones en el transcurso de la propia conversación y así poder adaptar el discurso en el momento en el que se está produciendo. Otros factores, como las exigencias curriculares, las limitaciones temporales y, en gran medida, una metodología de aprendizaje tradicional y automatizada, centrada a menudo en competencias gramaticales, se añaden a esta serie de obstáculos en la práctica diaria de la EO en FLE. Además, los escasos recursos digitales en el aula al alcance del alumno contribuyen a generar una enseñanza-aprendizaje convencional y en muchas ocasiones, mecánica, que se aleja de los objetivos actuales de adecuación a un mundo tecnológico. En este panorama de dificultades, la insuficiente presencia de las competencias sociolingüística y sociocultural en el aula de una LE provoca en el alumnado falta de conexión con la dimensión identitaria de la lengua y de su cultura.

En definitiva, la práctica docente del FLE encuentra en su camino una serie de escollos que dificultan el proceso de enseñanza-aprendizaje que podemos graduar en una escala, que recoge el nivel de afección en el desarrollo de la expresión oral en FLE, por orden de gradación, de mayor (en la base) a menor (en la punta), tal y como refleja el gráfico siguiente:

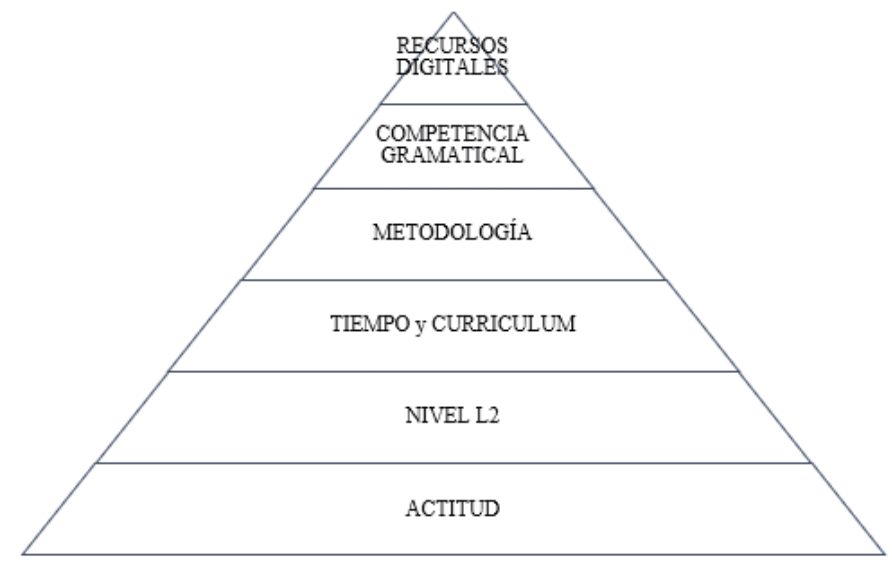

Gráfico 1: Pirámide del nivel de afección en el desarrollo de la EO en la práctica del FLE. Fuente: propia. 
Los problemas recogidos en esta pirámide confirman que nos encontramos ante un reto consabido en la comunidad educativa que nos presenta un doble desafío. Por un lado, mejorar las prácticas docentes de EO en el aula y, por otro, motivar un cambio de actitud entre el alumnado frente a la lengua francesa. Este reto, aunque evidente, no está resuelto a nuestro modo de ver, precisamente porque el proceso de enseñanza-aprendizaje se halla fuertemente asignaturizado por el sistema y esto dificulta enormemente el desarrollo de la expresión oral, tanto para el docente como para el alumno, que a menudo consideran esta competencia como un obstáculo casi imposible de gestionar en la práctica diaria de aula. Así lo confirma Montijano Cabrera (2003: 113), por ejemplo, tras llevar a cabo un sondeo en el que preguntaba a un número elevado de profesores de LE de la enseñanza secundaria, sobre las dificultades que encontraban a la hora de plantearse la enseñanza de la EO. La imagen dibujada, acerca de las posibilidades reales de alcanzar cotas de éxito en el desarrollo de esta destreza en contextos formales resultaba bastante pesimista. Entre los datos recogidos, tres elementos determinaban el bajo índice en las respuestas: la dificultad metodológica que implica la EO en el aula, la inhibición de los alumnos y su falta de motivación. En realidad, lo que revela esta situación es una cuestión de actitud, tanto del docente como del alumno. El profesor, por el tipo de metodología empleada, y el alumno, por el sentimiento que experimenta ante este tipo de actividad en el aula.

Por todo ello, en esta pirámide de complejidades, consideramos que la principal traba con la que se encuentra el docente de FLE reside en la que, a nuestro juicio, se halla en la base del sistema, es decir, en la actitud del alumnado ante el aprendizaje de la LF, ya que considera esta lengua un mero trámite para alcanzar su objetivo, que no es el aprendizaje real de una nueva LE, sino un requisito impuesto por el sistema para alcanzar su propósito, es decir su titulación final. Por tanto, se podría afirmar que, debido a este condicionante, el alumno no integra la lengua francesa como parte del ciclo general de su aprendizaje y la mantiene desinstalada de su proceso académico considerándola un trámite del mismo.

Con el fin de compensar estos obstáculos y para lograr mayor éxito en el desarrollo de la EO, mayor calidad para la enseñanza del FLE y mejor imagen de esta lengua entre el alumnado que la cursa como LE2 obligatoria, presentamos, en este trabajo, una experiencia docente llevada a cabo en el aula de FLE. Con ello, se trata de designaturizar la lengua francesa de tal manera que el alumno adopte una perspectiva interaccionista entre él y su lengua de aprendizaje, para que, lejos de alienarse de ella, se apropie de la lengua y consiga integrarla realmente en su proceso de aprendizaje. En este sentido, consideramos que el acercamiento a la comunidad hablante de lengua francesa, así como un profundo conocimiento de esta sociedad podría generar una imagen más favorecedora y alentadora para su porvenir profesional. El propósito, con este tipo de práctica docente, consiste en actuar desde la base de la pirámide ya que, solo desde ahí, se podrá generar un cambio en las actitudes del aprendiente y los resultados incidirán directamente tanto en el éxito 
del proceso como en la percepción del alumnado ante la lengua francesa. Si analizamos los componentes de la actitud enunciados por Rosenberg y Hovland (1960), comprobamos que efectivamente, el núcleo de la problemática planteada se encuentra en la actitud pues está compuesta por tres elementos que determinan el resultado de cualquier proceso: el componente cognitivo, relacionado con las ideas, percepciones y creencias, el componente afectivo, relacionado con los sentimientos y el componente conativo, es decir, de disposición para la acción. La suma de todos ellos (ideas + sentimientos + acciones) se traduce en la actitud que el individuo muestra hacia un objeto, una persona, una situación... Esto tres componentes indican claramente la interrelación entre emoción y razón lo que confirma que el estado emocional de un individuo determina su estilo cognitivo y, en consecuencia, caracteriza su actuación en aquella emoción.

Por tal motivo, si se modifica la actitud se podrá lograr un resultado distinto al hasta ahora obtenido durante este tipo de actividades en el aula. Así, la línea de actuación para diseñar el proyecto debe incidir directamente en modificar las ideas, sentimientos y acciones del alumnado durante el proceso de tal manera que se pueda provocar un cambio en sus actitudes. Además, como afirma Pearce (1982), para generar un cambio actitudinal en el alumnado, resulta esencial poseer información. Este autor observó que el hecho de contar con más información sobre un país concreto, su cultura y un mayor contacto intercultural conducía a actitudes más positivas hacia su lengua. Por lo que será necesario incluir este aspecto en el diseño del proyecto. Otro factor relacionado directamente con el cambio de actitud se encuentra en el interés personal del individuo, tal y como afirman Boninger, Brosnick y Berent (1995). Resulta evidente que cuanto mayor sea el interés del individuo ante el objeto de aprendizaje mayor será el éxito en el resultado y, en este sentido, cualquier alumno de FLE con alto interés personal obtendrá una mejoría sustancial en su proceso de aprendizaje. Dörnyei (2000) asegura que para lograr el deseado cambio actitudinal es necesario actuar desde dos ángulos característicos de los contextos de aprendizaje, el profesorado y los compañeros. Es evidente que el primero es el responsable de guiar al alumnado a ese cambio actitudinal, aportando información pertinente al proceso y una estrecha relación de trabajo con los segundos, generará ese interés personal en el alumno de cara al trabajo.

Por esta razón decidimos emplear una metodología de aprendizaje colaborativo y experiencial donde la intervención grupal entre compañeros como unidad social pudiera incidir positivamente en ellos y aumentar su interés personal en la tarea. En cuanto a los métodos de enseñanza con participación del alumno, donde la responsabilidad del aprendizaje depende directamente de su actividad, de su implicación y compromiso, coincidimos con Fernández March (2006: 42) en concluir que el proceso debe resultar más formativo que informativo de manera que se genere un aprendizaje más profundo, significativo y duradero. Cuando el alumno aborda el conocimiento de manera autónoma y experiencial, aspecto crucial para desarrollar las estrategias fundamentales que las nuevas generaciones de aprendientes nece- 
sitarán en su desarrollo profesional y personal, logra desarrollar un pensamiento más crítico y, en consecuencia, consigue mayor interés y motivación ante la tarea. Esta autora (2006: 43) asegura que un aprendizaje de estas características demanda metodologías que propicien la reflexión sobre lo que se hace, cómo se hace y qué resultados se logran, ya que de esta manera el alumno será capaz de utilizarlo como estrategia de mejora de su propio desempeño, desarrollando además la competencia más compleja de todas: la de aprender a aprender con sentido crítico sobre su actuación.

El hecho de disponer de información implica necesariamente ampliar el conocimiento con respecto a la lengua francesa, su cultura y sus comunidades hablantes en todo el mundo. En este sentido, la Francofonía ofrece el mayor centro de información al respecto. Sin embargo, el tratamiento que la Francofonía recibe en muchas ocasiones en el aula de FLE resulta desenfocado o limitado. Si atendemos a la investigación realizada por Barbier Muller (2017), observamos que el trato que esta recibe en los libros de textos utilizados en clase de FLE se halla lejos de representar lo que realmente significa. Por ello, consideramos absolutamente imprescindible centrarse en los recursos digitales para acceder a esta información bajo el soporte del uso de las TIC. En primer lugar, por la necesaria conexión entre la tecnología y el alumnado actual que encuentra en el uso de los recursos digitales su manera de relacionarse, de expresarse y de comprender su realidad, por tanto, de proyectarla. En segundo lugar, porque la capacidad de información acumulada en la Red y la inmediatez de su acceso posibilitan una mirada más crítica de cualquier cuestión. Los recursos disponibles en Internet sobre la Francofonía suponen un medio totalmente interactivo, global y electrónico que, en opinión de Crystal (2004: 88), tiene consecuencias directas en el aprendizaje pues a través de la Red, el alumno podrá entrar en contacto directamente con la competencia sociocultural de la realidad francesa y francófona. Durante el acercamiento a esta realidad, la conciencia y la comprensión de las relaciones entre la lengua materna del alumno y su segunda lengua extranjera (LM/L1+LE/L2) confluyen en el alumno y, esta conjunción, provoca la apropiación entre la LE2 y el alumno, es decir, entre su LM/L1 y su LE/L2 o, dicho de otro modo, entre el mundo del que se viene y el mundo de la comunidad de llegada. De esta manera, desarrolla una conciencia sociocultural e intercultural lo que le permitirá abrir el inmenso abanico de culturas formado por los universos de las dos lenguas, que se encuentran en medio del aprendizaje conjunto de la LM/L1+LE/L2 en el aula de FLE.

En consecuencia, este proyecto docente trata de aunar la interculturalidad que la Francofonía ofrece y la interconectividad que permite la Red, toda vez que se ocupa del aprendizaje de la expresión oral en lengua francesa mediante una metodología basada en los postulados propios del aprendizaje colaborativo. Según Srtijbos, Martens y Jochems (2004), el diseño de actividades basadas en el aprendizaje colaborativo debe atender necesariamente y de manera conjunta al tipo de tarea, el nivel de estructura de las actividades, el tamaño del grupo y la tecnología utilizada y, todo ello, deberá ser evaluado pues la 
evaluación debe formar parte del proceso indiscutiblemente. Para lograr la consecución de estos factores, la figura del docente resulta clave en todo este proceso, pues solo a partir del diseño y planificación del proyecto, de una correcta guía, motivación y ayuda al alumnado durante el proceso y una adecuada evaluación de la actividad, se podrá alcanzar el aprendizaje real tanto de contenidos como de desarrollo personal y, con ello, la mejora de sus competencias.

\section{Proyecto docente en el aula de FLE}

Siguiendo pues todas las premisas anteriores, hemos diseñado un proyecto docente llevado a cabo con alumnos universitarios de tercer y cuarto curso de Grado con nivel B1-B2 en el marco de la asignatura: Segundo Idioma Moderno (francés). La actividad planteada consiste en presentar grupalmente un exposé oral sobre un aspecto relacionado con la Francofonía.

El origen de nuestro proyecto docente se encuentra en la observación de nuestra práctica diaria docente. De manera general, constatamos que los alumnos muestran, por un lado, un rechazo importante a la participación oral en el aula y, por otro, otorgan poca utilidad a la lengua francesa para su porvenir laboral. En definitiva, dos cuestiones que dificultan considerablemente el proceso de enseñanza-aprendizaje. Por ello, cuando nos planteamos este proyecto, el reto era doble: fomentar la expresión oral en FLE del alumnado utilizando como contenido el mundo de la Francofonía y así abrir al alumno todas las posibilidades que la lengua y la cultura francesas pueden ofrecerle para su inserción en el mundo profesional. En este sentido, las Tic nos ofrecían el soporte perfecto para obtener información, a escala mundial de manera rápida y actualizada, acerca de la Francofonía, que representa el marco incomparable de la dimensión de apertura que supone el dominio de la lengua francesa. Para cuantificar la percepción del alumnado al respecto de estas cuestiones, decidimos proponerles un formulario de preguntas y así poder evaluar su opinión de manera previa al trabajo. Esto nos facilitaría el diseño de la tarea y los objetivos que nos debíamos proponer. Este mismo formulario se entregaría tras la finalización del trabajo con el propósito de comparar los resultados y valorar en qué medida la percepción del alumnado se había modificado.

El proyecto se dividía en cuatro fases: análisis, planificación, ejecución y evaluación tal y como se recoge en el siguiente gráfico: 


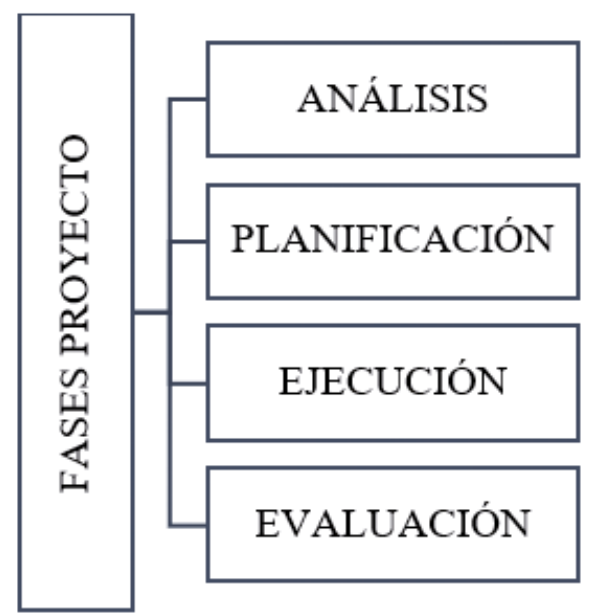

Gráfico 2: Fases del proyecto docente.

Fuente: propia.

En la primera fase, el análisis, nos planteamos dos cuestiones. La primera, establecer cuáles debían ser los requisitos previos que contribuirían a llevar a cabo posteriormente el proyecto en las mejores condiciones de aprendizaje. La segunda, analizar las dificultades que plantean los habituales contextos de enseñanza y aprendizaje en cuanto al desarrollo de la EO en el aula, con el fin de reflexionar acerca de las posibles soluciones al respecto. La correcta formulación de ambos aspectos aseguraría una adecuada planificación de la actividad, su correcta puesta en marcha, así como la obtención de unos resultados satisfactorios. El gráfico siguiente recoge los elementos básicos de la fase de análisis:

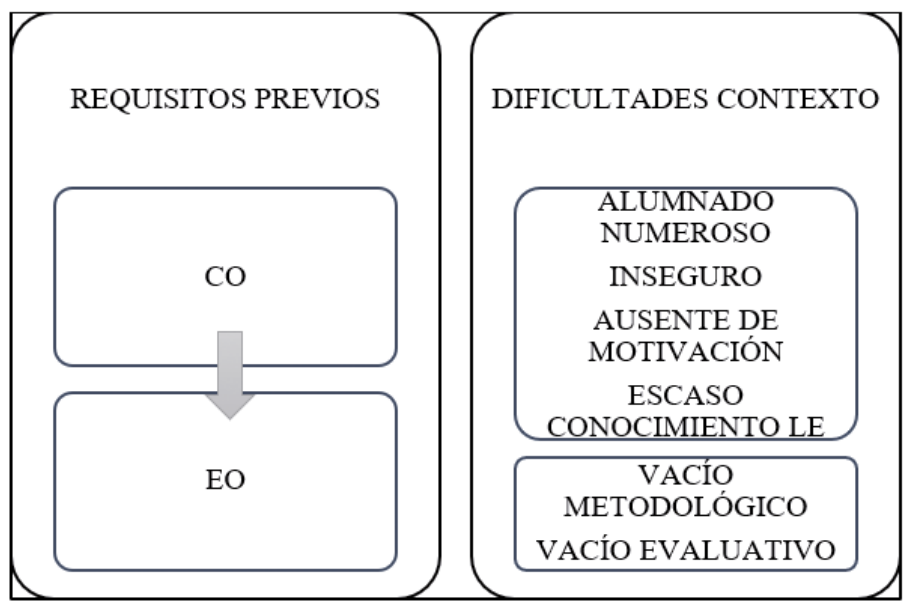

Gráfico 3: Fase de análisis.

Fuente: propia. 
Analizando este gráfico, en cuanto a los requisitos previos necesarios, resulta imprescindible que el alumnado conozca y maneje el contexto de la Francofonía para poder abordar posteriormente un trabajo de investigación y exposición sobre ella. De esta manera, el alumno se implicará en la actividad de expresión oral con mayor atención y alcance. Para ello, es fundamental acercar el mundo francófono al alumno, que, en muchas ocasiones, desconoce o ha profundizado poco en el mismo. En este sentido, la comprensión oral (CO) se presenta como la competencia más adecuada para introducir al alumnado en el universo de la Francofonía, precisamente porque, en toda capacidad comunicativa, la $\mathrm{CO}$ es condición sine qua non y anterior a cualquier desarrollo de la EO. Esto ya ocurre durante el normal desarrollo de la LM/L1, donde el individuo necesita un período extenso de comprensión de la lengua antes de formular las primeras palabras. La relación, por tanto, de necesaria dependencia entre ambas habilidades lingüísticas CO-EO en la LM/L1 también es aplicable en el aprendizaje de LE. Esta afirmación se halla fundamentada tanto por las teorías psicolingüísticas de adquisición de la lengua materna (ALM) como por las teorías de adquisición de segundas lenguas (ASL). Ambas confirman que el proceso de aprendizaje de una LE comparte muchos rasgos propios de la adquisición de la lengua materna (LM) y, por ello, los modelos cognitivos utilizados en este podrían resultar válidos igualmente para el aprendizaje de las LE. Martín Peris (2004: 262) lo explica por ejemplo cuando compara ambos procesos mentales, exponiendo que tanto uno como otro implican el desarrollo gradual del conocimiento de estructuras y rasgos lingüísticos a través de la aplicación de estrategias generales de percepción, almacenamiento y producción comunes a todo tipo de aprendizaje de lenguas.

Partiendo de las teorías lingüísticas de ALM, específicamente en la corriente cognitivo-constructivista del Desarrollo Intelectual de Piaget (1978: 8), sabemos que el individuo pasa necesariamente por un período mínimo de un año y que se extiende hasta los dos años, en el que obtiene el conocimiento de la lengua de manera pasiva, es decir, donde solo está interviniendo la adquisición de la competencia comprensiva oral de la lengua, anterior y necesaria a la aparición de su expresión oral. Sin esa evolución previa, la articulación de palabras comprensibles en una lengua y, por tanto, de la capacidad de expresarse oralmente en LM, ve mermadas sus posibilidades de éxito. Un claro ejemplo de ausencia de desarrollo de la expresión oral por falta de contacto y aprendizaje pasivo de comprensión lingüística oral se encuentra en los casos de hipoacusia prelocutiva, donde el niño no desarrolla el lenguaje hablado debido a la existencia de una grave alteración de la audición. Según Ruytens y cols (2006), el lenguaje hablado se procesa porque primero se produce una activación del área auditiva primaria y posteriormente se hace un análisis fonético. Como expresan Narbona y Chevrie-Muller (2001), para que se produzca el lenguaje hablado son imprescindibles tres componentes: la atención selectiva, la percepción categórica de los sonidos del habla y la recepción auditiva del habla. Por consiguiente, podemos afirmar que la CO en una lengua es requisito anterior y sine qua non para desarrollar una correcta EO en esa lengua. 
Si atendemos a las teorías psicolingüísticas de ASL, asimismo observamos que numerosos autores afirman que el proceso de aprendizaje de segundas lenguas (SL) se rige por las mismas leyes que el de las LM, sosteniendo así la hipótesis de la identidad de Wode (1974). De hecho, entre las teorías innatistas, basadas en los postulados del Enfoque Natural de Chomsky, Krashen y Terrell (1983), afirman que la adquisición de una lengua solo puede ser alcanzada por la comprensión de mensajes. Este enfoque ve ambos aprendizajes como similares. También encontramos apoyo en las teorías interaccionistas de ASL, por cuanto sostienen que la interacción comunicativa entre los hablantes, en las que intervienen CO-EO, es determinante para la adquisición de segundas lenguas. Por consiguiente, también desde este contexto, se confirma la necesaria adquisición previa de la $\mathrm{CO}$ para acceder a la EO en el proceso de aprendizaje de LE. En definitiva, a la hora de plantear una actividad lingüística de EO en la que el contenido gire en torno a la Francofonía, es crucial que previamente el alumno haya abordado en profundidad este tema con ejercicios de comprensión oral. De esta manera, la escucha activa le permitirá conocer y/o ampliar sus conocimientos sobre el mundo francófono, lo que sin duda contribuirá a una mejora sustancial en el momento de reportar sus propios contenidos. Estas actividades deberán producirse durante un período prolongado para que la asimilación sea sustancial y significativa y repercuta favorablemente en la producción oral del alumno posteriormente.

Siguiendo el gráfico 3, en lo que se refiere a las dificultades del contexto, Rabeá (2013: 9-15) asegura que es habitual encontrarse durante la práctica docente limitaciones de conocimiento de la LE, presión de tiempo para realizar la tarea, participación desigual de los alumnos, inhibición o sentimiento de vergüenza, miedo a cometer errores, dificultad para mantener la lengua extranjera en el transcurso del intercambio comunicativo, falta de confianza en uno mismo o falta de interés real en el tema del que se trata, entre otros. De hecho, en nuestra práctica docente habitual observamos estos condicionamientos que acarrean como consecuencia lo que afirma Young (2000: 79), es decir, que la ansiedad, ante el aprendizaje de una lengua, está negativamente relacionada de manera especial con destrezas orales. Además de estos factores que influyen de manera negativa para plantear actividades de expresión oral en el aula, se da la circunstancia de que muy a menudo el alumnado suele ser numeroso lo que dificulta todavía más esta cuestión. Por último, existen otros dos obstáculos dentro del contexto: el vacío metodológico y del vacío evaluativo cuyo tratamiento se verá en las fases de planificación y de evaluación respectivamente.

Una vez finalizada la fase de análisis, debíamos planificar la actividad. Esta fase se divide en dos partes: planificación I, que consiste en diseñar una o varias propuestas para tratar de resolver las dificultades del contexto, de tal manera que se minimice el impacto de los posibles resultados negativos durante la ejecución del proyecto; y planificación II, donde se presenta el proyecto a los alumnos y se detallan todas sus características y requisitos. La fase de planificación queda recogida en el siguiente gráfico: 


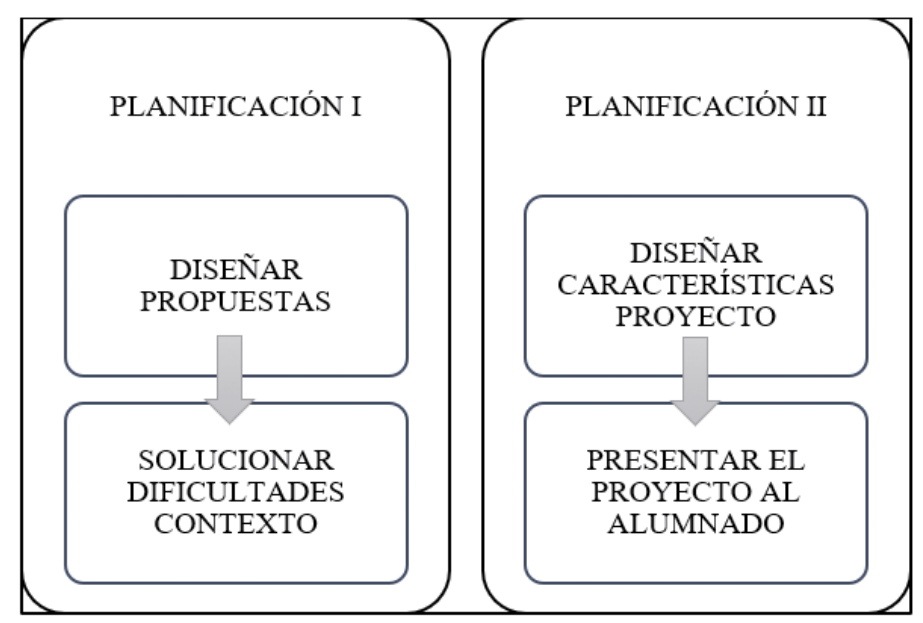

Gráfico 4: Fase de planificación.

Fuente: propia.

En la primera parte de la planificación, hay que tratar de solventar todos los elementos del contexto identificados como desfavorecedores. Para ello, es crucial centrar el diseño del proyecto en el alumno, a través de un aprendizaje colaborativo y experiencial. Las experiencias colectivas del trabajo en grupos resultan idóneas para conseguir mayores estímulos en el desempeño de la tarea. Bourgeois (1997: 225) sostiene que, gracias al aprendizaje cooperativo, los estudiantes en grupos suficientemente compactos son capaces de realizar el trabajo con un alto grado de motivación pues carecen de supervisión directa y unilateral del profesor. En este sentido, conviene conformar grupos de trabajo heterogéneos por un número impar de miembros con funciones y responsabilidades específicas (PROSIEC, 20007: 231). En la misma línea, Barbero (1996) señala que las actividades compartidas en grupo requieren acuerdos, disposición pausada, tono colectivo y atención a las voces de los demás. El apoyo entre los miembros del grupo sin duda contribuye a reforzar aquellos aspectos de inhibición e inseguridad que experimentan los alumnos ante actividades participativas. Por otro lado, la falta de seguridad ante la limitación de conocimientos se ve soslayada por el aprendizaje previo adquirido mediante las actividades de comprensión oral realizadas a lo largo de las sesiones durante el cuatrimestre. La inmersión en el mundo francófono y el trabajo directo llevado a cabo durante el tiempo de aula previo a la presentación del proyecto supone un bagaje importante de conocimiento de la materia, que contribuye de manera muy positiva para exponer en óptimas condiciones. Por todo ello, el aprendizaje colaborativo y experiencial reúne importantes características para solventar varias de las dificultades identificadas en el contexto, tales como la falta de motivación y de seguridad frente a la práctica individual de actividades de EO en el aula.

En cuanto al vacío metodológico que caracterizan este tipo de actividades, las meto- 
dologías activas suponen una estrategia determinante ya que conciben el aprendizaje como un proceso constructivo y no receptivo. Glaser (1991) explica que la psicología cognitiva ha mostrado consistentemente, que una de las estructuras más importante de la memoria es su estructura asociativa. El conocimiento, al estar estructurado en redes de conceptos relacionados, que se denominan redes semánticas, permite que la nueva información se acople a la red ya existente. Dependiendo de cómo se realice esta conexión, la nueva información puede ser utilizada o no, para resolver problemas o reconocer situaciones. Esto implica la concepción del aprendizaje como proceso y no únicamente como una recepción y acumulación de información. En consecuencia, se trata de que el alumno investigue y estudie su propia materia de aprendizaje y construya su propio conocimiento porque de esa manera se asegurará el aprendizaje. Además, las metodologías activas enfatizan que la enseñanza debe tener lugar en el contexto de problemas del mundo real o de la práctica profesional. Por ello, deben trabajar con contenidos lo más cercanos posibles al contexto profesional en que el estudiante se desarrollará en el futuro. La contextualización de la enseñanza promueve la actitud positiva de los estudiantes hacia el aprendizaje y su motivación, lo que es imprescindible para un aprendizaje con comprensión. Por ello, se trata de acercar la Francofonía al alumno y que sea él el constructor de ese conocimiento. De esta manera, se contextualiza su aprendizaje generando una actitud favorable hacia la lengua francesa y las posibilidades profesionales que le puede ofrecer. Se trata de provocar en el alumno lo que Labrador y Andreu (2008: 5) proponen cuando definen las metodologías activas como aquellos métodos, técnicas y estrategias que utiliza el docente para convertir el proceso de enseñanza en actividades que fomenten la participación del estudiante y le lleven al aprendizaje.

En la segunda parte de la planificación, se presenta el proyecto a los alumnos, se describen las características del proyecto y el contexto de la actividad. Se propone al alumnado que decidan la composición de los grupos y elijan los temas de trabajo que deberán ser aprobados por el profesor.

Contexto de la actividad de EO:

- descripción: presentación de una exposición oral sobre un tema relacionado con la Francofonía.

- objetivos:

o didácticos: comprender la dimensión de la lengua y de la cultura francesas en el panorama internacional

o comunicativos: expresar oralmente en lengua francesa

o personales: apreciar la importancia de la lengua francesa en el mercado laboral

- contenidos: Francofonía (cada grupo elige un tema concreto)

- metodología: aprendizaje colaborativa y experiencial y metodologías activas.

- recursos: digitales (Internet-PPT- Prezi-Formularios de Google) 
- grupos de trabajo: 3 alumnos/grupo

- período: un cuatrimestre

- tiempo exposición/alumno: $5 \mathrm{~min}$

- $\quad$ sistema de evaluación: inicial, continua y final

- tipo de evaluación: heteroevaluación y coevaluación

- criterios de evaluación: rúbrica de exposición oral.

Las dos siguientes fases, la fase de ejecución y la de evaluación, se desarrollan de manera conjunta. Mientras los alumnos presentan sus trabajos oralmente, sus compañeros evalúan sus presentaciones con la rúbrica de exposición oral. Al mismo tiempo, el profesor evalúa cada presentación con la misma rúbrica. Durante sus presentaciones utilizaron recursos TIC, concretamente Internet como fuente de información y los programas PPT y Prezi como programas de presentación. El uso de estas tecnologías potencia el aprendizaje de forma colaborativa ya que permiten a los alumnos trabajar de forma asíncrona y distante además de la rapidez y gran variedad de información a su disposición. Es innegable el poder que ejercen las nuevas tecnologías y por ello coincidimos con Calzadilla (2002: 8) en que el aprendizaje colaborativo se beneficia de las nuevas tecnologías por los estímulos de comunicación interpersonal que se dan en el aprendizaje virtual ya que posibilita el intercambio de información de los miembros del grupo involucrado y refuerza la interactividad entre ellos y su trabajo. Las posibilidades que este tipo de programas ofrecen, como pueden ser insertar vídeos, diseñar diapositivas, realizar transiciones o animaciones, aumentan considerablemente la motivación del alumnado ante su trabajo. En definitiva, como expresan Zañartu Correa (2000); Martínez Cabrera et al. (2004); Amor, Fuentes, Jiménez y Pinto (2004), a través de la técnica, logramos que las características del aprendizaje colaborativo sean más efectivas y, por consiguiente, más significativo.

Un rasgo común y esencial de todas las metodologías activas es el papel de la evaluación. El vacío evaluativo que suele acompañar tradicionalmente a las actividades de EO en el aula provoca que el alumnado se enfrente a ellas con cierto recelo pues, a menudo, considera que se trata de una evaluación meramente subjetiva por parte del docente, en lugar de medir el trabajo bajo una perspectiva objetiva, concretizada y, en consecuencia, más justa. Morales y Landa (2004: 155) explican hasta qué punto es importante tener en cuenta el aporte individual, así como el producto final generado por el grupo. La evaluación es un seguimiento continuo y sistemático que se le hace para identificar los logros y las dificultades presentadas en el proceso y poder tomar decisiones que lleven a un mejoramiento de la calidad educativa. Todo ello implica, como afirman Castillo Arredondo y Cabrerizo Diago (2010: 19) que el proceso evaluativo no se reduce al hecho habitual de evaluar solo los contenidos, sino que también debe tener en cuenta distintos aspectos que intervienen en el proceso de educativo de los alumnos: las habilidades, las actitudes y valores, y las estrategias de aprendizaje, sin 
olvidar los aspectos docentes del proceso de enseñanza que inciden en el aprendizaje: metodología empleada, intercomunicación en el aula, nivel de exigencia, etc. Para evaluar con estas características, es necesario considerar distintos tipos y sistemas de evaluación de manera que los alumnos aprendan a evaluar a sus compañeros, a sí mismos y al producto, es decir al proyecto planteado por el profesor. Así, podrán valorar e identificar qué aspectos podrían mejorarse en sucesivas ocasiones y el aprendizaje sea lo más significativo posible. Bajo estas premisas, nuestro proyecto propone un sistema triple de evaluación:

- Evaluación inicial o diagnóstica, para verificar el nivel de preparación y conocimientos de los alumnos y su opinión sobre la lengua francesa y la Francofonía, así como el tipo de metodología con el que se va a trabajar. Para ello, se utilizan los formularios Google que proporcionan agilidad al proceso además de continuar en la línea de introducir en el proceso de aprendizaje las TIC. Esta evaluación tiene lugar en la fase de planificación II, antes de presentar el trabajo a los alumnos.

- Evaluación continua, donde el alumnado y el profesor evalúan a los estudiantes que presentan su trabajo gracias a la rúbrica de evaluación de EO; Delgado y Oliver (2006) afirma que la evaluación continua tiene por objetivo la valoración del grado de aprendizaje conseguido por el educando y adquiere una nueva dimensión al girar el aprendizaje en torno a él. En este sentido, debe estar correctamente diseñada para valorar si el estudiante ha alcanzado el objetivo, no sólo los conocimientos sino también las competencias previamente definidas por el educador para una materia concreta. Por ello, resulta muy beneficiosa para el estudiante pues le permite evaluar el trabajo de sus compañeros, algo en lo que implícitamente le permite reconocerse y valorar su propio trabajo. Esta evaluación se realiza de manera bidireccional, un sentido descendente (profesor-alumno) y otro lateral (alumno-alumno). En el primer caso, se trata de una heteroevaluación, en el segundo, de una coevaluación. Las rúbricas de evaluación empleadas en ambos casos resultan la herramienta idónea ya que, a través de ellas, tanto los alumnos como los docentes conocen a priori las expectativas requeridas y los criterios de desempeño o de productos a evaluar, así como los distintos niveles de calidad o logro en ellos.

- Evaluación final, donde el alumnado debe evaluar el resultado del proyecto. Con esto, se valora el criterio del profesor por el tipo de proyecto planteado y la metodología utilizada. Además, se evalúa de nuevo la opinión del alumnado de cara a la lengua francesa y así comprobar si ha habido modificación en la actitud del alumnado frente a esta LE de aprendizaje. Para ello, se utiliza otro formulario Google con una serie de preguntas al respecto de estas cuestiones. Esta evaluación tiene un sentido ascendente (alumno-profesor) y se trata de una heteroevaluación. 
El gráfico siguiente recoge todo el proceso evaluativo:

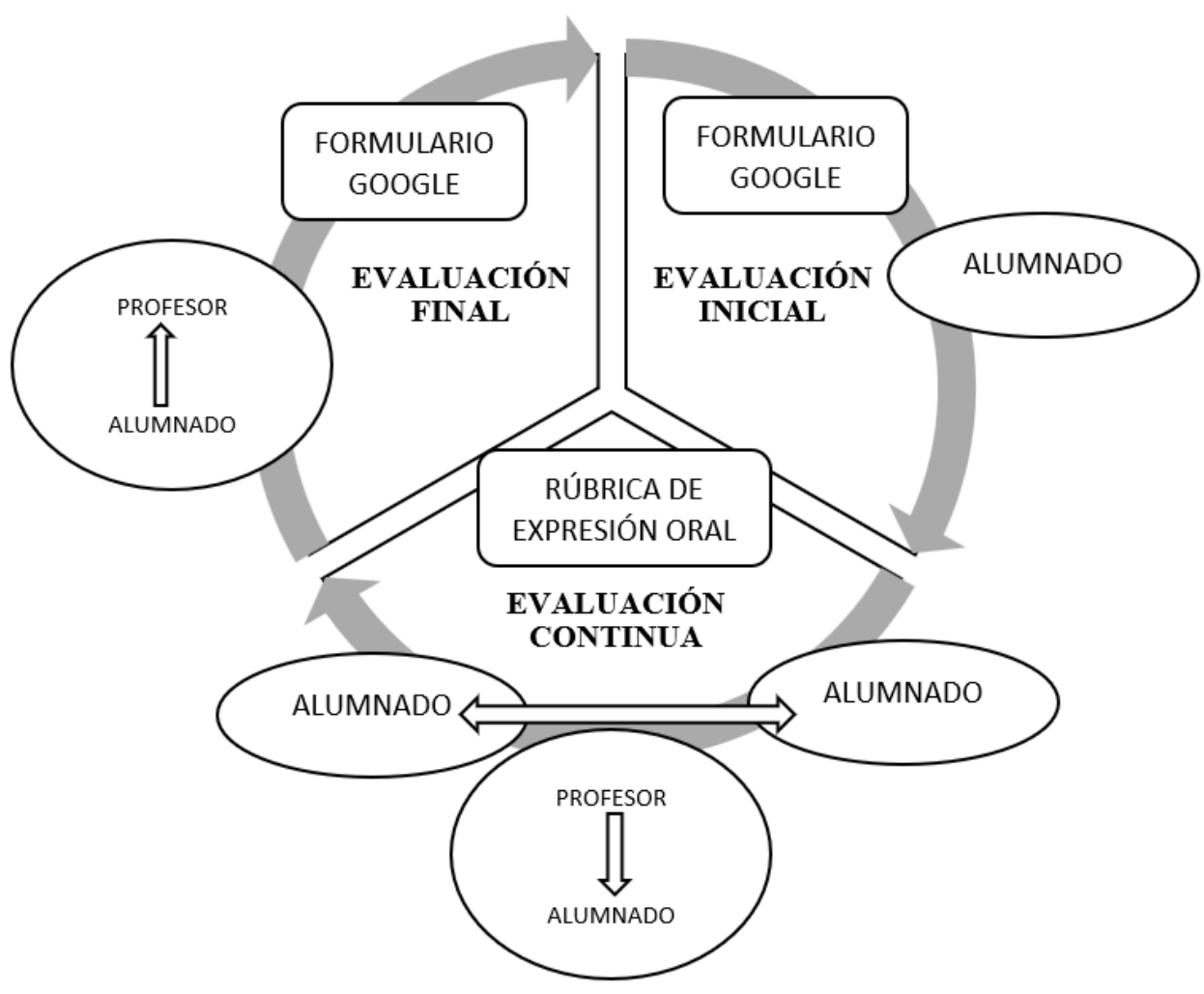

Gráfico 5: Proceso de evaluación.

Fuente: propia.

En resumen, la evaluación resulta parte fundamental del proceso pues gracias a un tipo de evaluación formativa y no sumativa, el alumno que puede conseguir que su aprendizaje sea realmente significativo. El objetivo consiste en lograr una evaluación para el aprendizaje o el llamado assessment for learning, propuesto por Stiggings (2014), quien afirma que la intención de la evaluación pasa por involucrar a los estudiantes en la misma como mecanismo de autorregulación. La evaluación de tendencia formativa incide directamente en el incremento de la aproximación de la acción del profesor (enseñanza) a los logros de los alumnos (aprendizaje), lo que podría traducirse en un mayor logro académico, estándares más altos y un conocimiento más profundo. Este tipo de evaluación se centra más en el contexto y en especial en el proceso de la enseñanza-aprendizaje. En contraste con la evaluación sumativa, de carácter generalmente final, la evaluación formativa se aplica en los distintos 
estadios del proceso de enseñanza-aprendizaje. Un uso adecuado de sus resultados, a través de un feedback inmediato, directo y permanente, se convierte en el mejor instrumento para asegurar la coherencia entre objetivos, proceso y producto de la enseñanza-aprendizaje y, en consecuencia, para su optimización y calidad. La evaluación para el aprendizaje, además de considerar los aspectos de una evaluación formativa, pone de relieve el papel activo del alumno durante todo el continuo para el logro de sus procesos autorregulativos, tanto individualmente como con sus pares (Popham, 2014; Stiggings, 2014). En definitiva, este tipo de evaluación, por sus características, resulta la herramienta idónea en el aprendizaje colaborativo y experiencial.

\section{Resultados}

Hemos medido los resultados del proyecto a través de los dos cuestionarios Google planteados tanto al inicio del proyecto como a su finalización. Los datos recogidos en ambos momentos se muestran en dos gráficos comparativos. El primero de ellos, con respecto a la opinión acerca de la lengua francesa y la Francofonía como ilustra el siguiente gráfico:

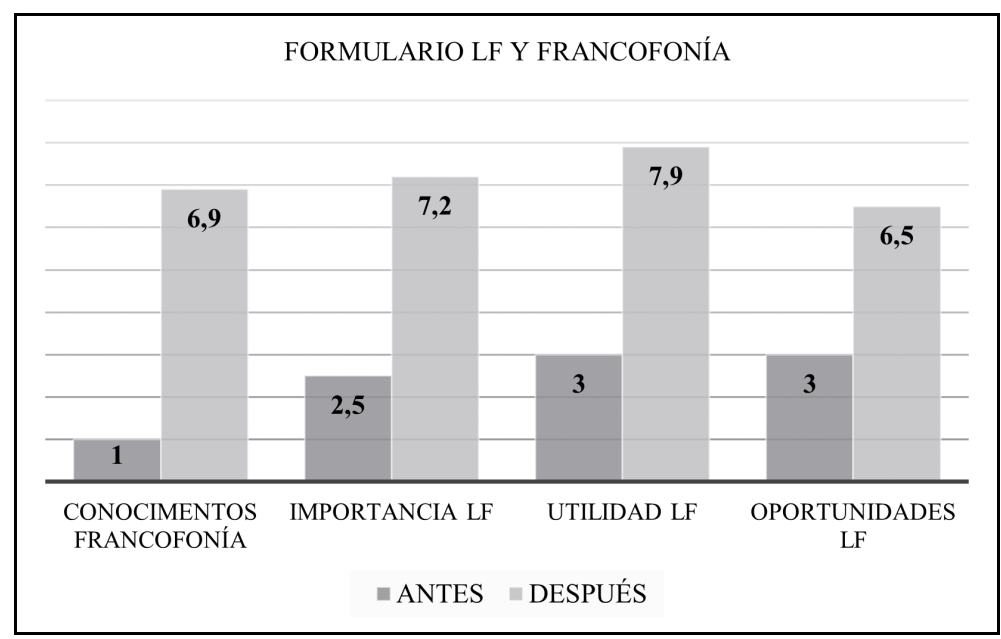

Gráfico 6: Resultados formulario sobre la lengua francesa (LF) y la Francofonía.

Fuente: propia.

En este gráfico se puede comprobar que el conocimiento del alumnado sobre el mundo francófono antes de iniciar el proyecto era prácticamente nulo, ya que tan solo el 1\% declara conocer algo al respecto. Al finalizar el trabajo, los alumnos afirman que han aumentado sus conocimientos hasta casi el $7 \%$. Con respecto a la importancia que el alumnado le otorgaba a la lengua francesa, la opinión varía sustancialmente pues se observa que, antes de 
la realización de este proyecto, la imagen que los alumnos tenían de esta lengua era pobre $(2,5 \%)$, sin embargo, tras el proyecto, su opinión cambia considerablemente alcanzando un $7,2 \%$. Lo mismo ocurre cuando se les pregunta acerca de la utilidad y oportunidades que esta lengua les puede facilitar en su acceso al mercado laboral. Los alumnos otorgaban a esta lengua una utilidad y unas oportunidades de un 3\% antes del inicio del trabajo y un 7,9\% y un $6,5 \%$ respectivamente a su finalización.

El segundo gráfico muestra los datos recogidos acerca de las opiniones del alumnado respecto de la metodología empleada en este proyecto:

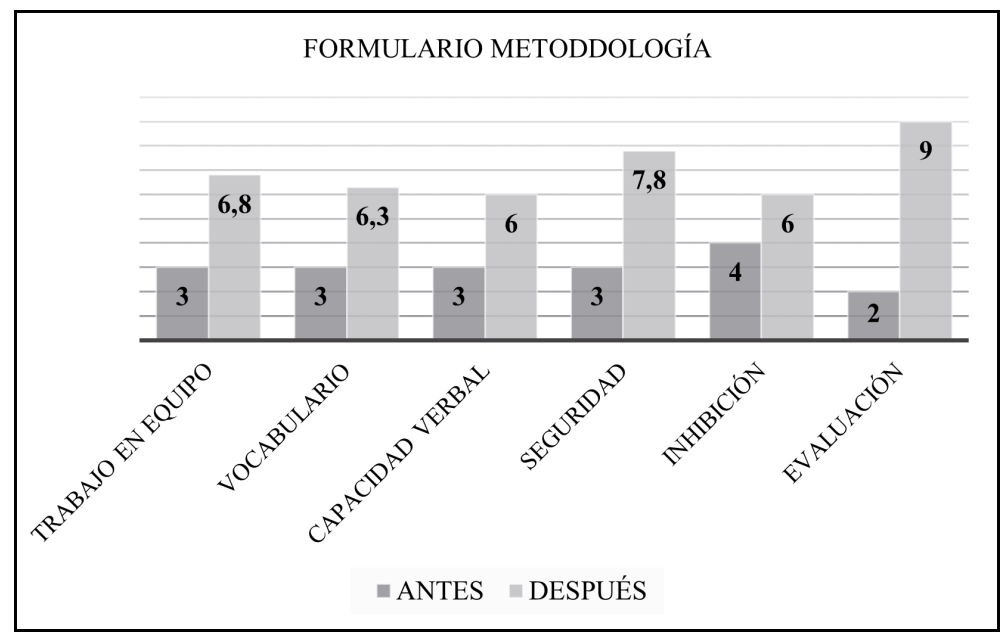

Gráfico 7: Resultados formulario sobre la metodología.

Fuente: propia.

Los datos recogidos en ambos momentos del proyecto muestran que, en todas las cuestiones, la apreciación del alumnado varía sustancialmente antes y después de su trabajo. Si observamos de manera general los porcentajes iniciales, las valoraciones oscilan entre el $2 \%$ (en cuanto a su valoración respecto del tipo de evaluación en las actividades de EO) y el 4\% (en lo que se refiere al sentimiento de inhibición que experimentan ante este tipo de tareas), siendo la mayoría de un 3\% (acerca de las opiniones sobre el trabajo en equipo, el vocabulario y la capacidad verbal adquiridos y la seguridad con la que se enfrentan a esta práctica en el aula). Sin embargo, los porcentajes finales revelan un incremento importante en la opinión del alumnado. Concretamente, aumentan hasta un $6 \%$ en cuanto a la inhibición y la capacidad verbal adquirida, un $6,3 \%$ en vocabulario aprendido y un $6,8 \%$ en valoración del trabajo en equipo. Las valoraciones más altas resultaron para la seguridad experimentada por el alumnado, en particular hasta un 7,8\% de subida y para la evaluación, que consiguió hasta un $9 \%$ de puntuación. 


\section{Conclusiones}

Los resultados obtenidos en este trabajo revelan conclusiones importantes y positivas para la práctica docente en FLE, especialmente en tres aspectos: la actitud del alumnado, el nivel de LE2 y la metodología. En lo que se refiere a la actitud del alumnado frente a la lengua francesa, gracias al enfoque adoptado en este proyecto, se ha logrado un cambio de actitud muy significativo, pues la imagen de su LE2 y de las oportunidades profesionales que esta le puede brindar han resultado muy favorecedoras con respecto a la percepción general inicial. El trabajo realizado ha contribuido eficazmente a una apertura en torno a esta lengua, donde la mirada actual se resulta más crítica y real y no mediatizada por condicionantes externos establecidos en la sociedad, donde al parecer la única lengua útil de aprendizaje resulta la LEI por excelencia. Los alumnos toman en consideración ahora la utilidad laboral y personal que esta lengua les puede reportar para su desarrollo profesional. Por otro, en lo que respecta al desarrollo personal y académico del alumnado, consideramos que sus habilidades orales han mejorado sustancialmente, así como sus competencias lingüísticas y discursivas, principalmente gracias al protagonismo que han adquirido durante su aprendizaje. A pesar de que existe la creencia generalizada de que la alteración de las actitudes negativas resulta complicada, un adecuado tratamiento de las actividades demuestra que es posible generar ese cambio. Es evidente pues que las actitudes y las estrategias de aprendizaje de la LE2 influyen en su proceso de aprendizaje, por lo que resulta esencial trabajar desde prácticas docentes que favorezcan la percepción del alumnado ante esa LE2. Y, en este sentido, el proyecto docente presentado ha contribuido significativamente a modificar esas actitudes negativas preconcebidas con las que el alumno iniciaba el proceso.

En cuanto al nivel de L2, la valoración final tan positiva del alumnado frente a los aprendizajes adquiridos durante el proceso contribuye al éxito del proyecto. Por ello, se confirma que lograr una elevada competencia en L2 influye de manera decisiva en la proyección de unas actitudes positivas hacia la L2 lo que, sin duda, beneficia y facilita el proceso de aprendizaje. Por tanto, se puede afirmar la existencia de una relación de causalidad entre el logro obtenido y la actitud mostrada, y ello a su vez, conduce a confirmar el efecto directo en la motivación y la seguridad del alumno en el desarrollo de la expresión oral en el aula de FLE.

Por último, la metodología utilizada en este proyecto, experiencial y colaborativa, donde el sistema de evaluación formativa es clave para su adecuada consecución, ha resultado también determinante en el éxito alcanzado. Se demuestra que los alumnos que desarrollan sus propias estrategias de aprendizaje, que analizan sus logros y fallos, que participan de la creación de su proceso de aprendizaje y al mismo tiempo se responsabilizan de él, consiguen modificar sus actitudes hasta convertirlas en favorecedoras para su LE2 de aprendizaje.

Como conclusión, la puesta en marcha y realización de un proyecto docente de expre- 
sión oral en el aula de FLE, en los que la Francofonía represente el eje central, el colaborativo el medio y las TIC el soporte, contribuye de manera muy eficaz al proceso de conversión en el alumno, que pasa de ser un alumno que adquiere simplemente conocimientos lingüísticos de la lengua francesa a convertirse en un alumno con conciencia interlingüística e intercultural respecto de esta lengua. En definitiva, los objetivos propuestos, la metodología utilizada y las conclusiones alcanzadas tras este proyecto revelan la viabilidad de un aprendizaje significativo para el alumno y aporta no solo calidad educativa para el sistema, sino lo que verdaderamente resulta determinante para el proceso de aprendizaje, la adquisición y mejora de su capacidad expresiva en lengua francesa, sin perder de vista aspectos como la consolidación de sus conocimientos, el acercamiento al universo francófono en toda su dimensión sin olvidar que contribuye a estimular su motivación e inhibición ante esta destreza lingüística en el aula de FLE.

\section{Referencias bibliográficas}

Auzen, Icek. 1988. Attitudes, Personality and Behaviour. Londres, Open University Press.

AlcobA, Santiago. 2000. La expresión oral. Barcelona, Ariel.

BARbero, Jose Ignacio. 1996. “Tratamiento pedagógico de lo corporal (TPC). Hacia la construcción de un discurso autónomo en/de la Educación Física" in Actas del III Congreso Nacional de Educación Física de Facultades de Educación y XIV de Escuelas Universitarias de Magisterio, 19-29. Universidad de Alcalá.

BArbier Muller, Clotilde. 2017. "La diversidad lingüística y cultural de la francofonía en clase de Francés Lengua Extranjera: ¿mito o realidad?” in Revista Fuentes Humanísticas, n. ${ }^{\circ}$ 28 (54).

Boninger, D.S.; Krosnick, J.A. \& M. K. Berent. 1995. "Origins of attitude importance: Self-interest, social identification, and value relevance" in Journal of Personality and Social Psychology, 68, 65-82.

BourgeoIs, Étienne. 1997. Interacciones sociales y aprendizaje. Niza, Andereg 3.

Calzadilla, María Eugenia. 2002. "Aprendizaje colaborativo y tecnologías de la información y comunicación” in Revista Iberoamericana de Educación, vol. 29 (1) 1-10. <https://doi. org/10.35362/rie2912868>.

Arredondo, Santiago Castillo y DIAGO, Jesús Cabrerizo. 2010. Evaluación educativa de aprendizajes y competencias. Pearson Educación.

CRYSTAL, David. 2004. La revolución del lenguaje. Alianza Editorial. Madrid.

Delgado, Ana María y Oliver, Rafael. 2006. "La evaluación continua en un nuevo escenario docente" en Revista de Universidad y Sociedad del Conocimiento, RUSC, (3)1, UOC.

DöRnYeI, Zoltán \& SchmidT, Richard. 2000. Motivation and Second Language Acquisition, Honolulu, University of Hawai'i, Second Language Teaching and Curriculum Center. 
Fernández March, Amparo. 2006. "Metodologías activas para la formación de competencias", en Educatio siglo XXI, (24) 35-36.

GLASER Robert. 1991. "The Maturing of the relationship between the science of learning and cognition and educational practice" in Learning and Instruction, (1)129-144. U.S.A., University of Pittsburgh.

Labrador, Maria José y Andreu, M. Ángeles. 2008. Metodologías activas. Grupo de innovación en metodologías activas (GIMA). Valencia, Editorial UPV.

Lasagabaster, David. 2003. Trilingüismo en la enseñanza. Actitudes hacia la lengua minoritaria, la mayoritaria y la extranjera. Lleida, Editorial Milenio.

MARTín Peris, Ernesto. 2004. “Qué significa trabajar en clase con tareas comunicativas?” in Revista electrónica de didácticalespañol lengua extranjera, red ELE, (0), 34-57.

Montijano Cabrera, M. ${ }^{a}$ del Pilar. "Un análisis de las aportaciones docentes y discentes con vistas a obtener una mayor efectividad en la enseñanza de la expresión oral en LE" in Barros García, P. et al. (eds.) Enseñanza de la Lengua III. Granada, Editorial Ave María, 111-22.

Morales, Patricia \& LANDA, Victoria. 2004. “Aprendizaje basado en problemas. ProblemBased Learning" in Theoria (13), 145-147.

Narbona, Juan y Chevrie-Muller, Claude. 2001. El lenguaje del niño. Barcelona, Masson, $2^{\mathrm{a}}$ ed.

Piaget, Jean. 1978. Formaçao do símbolo da criança. Río de Janeiro, Guanabara-Koogan.

PeArCe, Philip L. 1982. "Tourists and their hosts: some social and psychological effects of inter-cultural contact" in Cultures in contact. Studies in cross-cultural interaction, 199-221.

Prosiec. 2007. Proyecto Salesiano de Innovación Educativa y Curricular. Quito, Editorial Don Bosco.

Pophan, W. James. 2014. Classroom assessment. What teachers need to know. Nueva Jersey, Pearson.

RABÉA, Berraghda. 2010. "El desarrollo de la expresión oral en lengua extranjera". Universidad de Bouzaréah: <https://cvc.cervantes.es/ensenanza/biblioteca_ele/publicaciones_centros/PDF/argel_2010/02_rabea.pdf> [25/03/2021].

Richards, Jack C., Platt, John \& Heidi Platt. 1992. Diccionario de lingüistica aplicada y enseñanza de lenguas, versión española y adaptación de Carmen Muñoz Lahoz y Carmen Pérez Vidal. Barcelona, Ariel.

Rosenberg, Milton J. \& Hovland, Carl I. 1960. "Cognitive, affective, and behavioral components of attitudes" in Rosenberg, Milton J. et al. (eds.). 1960. Attitude Organization and Change: an Analysis of Consistency among Attitude Components. New Haven, Yale University Press.

Ruytens, L., Willemsem, A., Van Dijk P, Uit H, Albers F. Functional imaging of the auditory system using PET. Acta Otolaryngol. (126) 1236-44, en IzQUIERDO, Marcos Rossi y Labella Caballero, Torcuato. Audición y Lenguaje. El niño sordo. Hospital Clínico Universitario de Santiago de Compostela, (31), A Coruña. 
Anales de Filología Francesa, n. ${ }^{\circ}$ 29, 2021

M. ${ }^{a}$ DOLORES ASENSIO FERREIRO

Stiggins, Richard. 2014. Revolutionize assessment. Empower students, inpire learning. Thousand Oaks, CA, Corwing.

Strijbos, J. Martens, R. L. \& Jochems, W. M. G. 2004. "Designing for Interaction: Six Steps to Designing Computer-Supported Group-Based Learning" in Computers \& Education, $42,403-424$.

Young, T. 1995. "Professional development for immersion teachers in Finland" in Buss, M. \& Lauren, C. (eds.) Language teac. Vaasa, Publicaciones de la Universidad.

ZaÑartu Correa, Luz María. 2000. “Aprendizaje colaborativo: una nueva forma de diálogo interpersonal en red" in Revista digital de educación y nuevas tecnologías. Contexto Educativo, 28. 
